\title{
Development of magnetic navigation method based on distributed control system using magnetic and geometric landmarks
}

\author{
Naoki Akai ${ }^{*}$, Sam Ann Rahok ${ }^{2}$, Kazumichi Inoue ${ }^{1}$ and Koichi Ozaki ${ }^{1}$
}

\begin{abstract}
Background: In order for a robot to autonomously run in outdoor environments, a robust and stable navigation method is necessary. Especially, to run in real-world environments, robustness against moving objects is important since many pedestrians and bicycles come and go. Magnetic field, which is not influenced by the moving objects, is considered to be an effective information for autonomous navigation.

Methods: Localization technique using a magnetic map, which records ambient magnetic field, has been proposed. The magnetic map is expressed as a linear map. When using this linear magnetic map, swerving from the desired path is a fatal problem. It is because that the magnetic map contains only magnetic data on a desired path. In the paper, we propose a novel navigation method which allows a robot to precisely navigate on a desired path even if localization is performed on the basis of the linear magnetic map. The navigation is performed by using a control method based on a DCS (Distributed Control System). In the system, several navigation modules are executed in parallel, and they independently control the robot by using magnetic and geometric landmarks.

Results and discussion: We conducted three navigation experiments. Our robot could perfectly accomplish all navigation even if it was disturbed by many moving objects during the navigation.

Conclusions: The control method based on the DCS could switch the navigation module for controlling the robot to cope against the change of its surroundings. The precise and robust navigation was achieved with the proposed method.
\end{abstract}

Keywords: Magnetic navigation; Distributed control system; Mobile robots for public space; Autonomous navigation

\section{Background}

An autonomous mobile robot can be used as a service robot in various fields such as transportation, cleaning, and guiding. To realize these services, a robust and stable navigation method is necessary.

Autonomous navigation methods using artificial landmarks (e.g. magnetic tapes or makers) have been proposed [1]. In some factories, automated guided vehicles traveling on magnetic tapes are practically used. However, using such a system requires environmental arrangement.

In contrast, autonomous navigation methods, which do not depend on artificial landmarks, have been recently

\footnotetext{
*Correspondence: akai@ir.ics.utsunomiya-u.ac.jp

1 Department of Innovation System Engineering, 7-1-2, Yoto, Utsunomiya-shi, Tochig, Japan
}

Full list of author information is available at the end of the article proposed. For indoor navigation methods, the accuracy have been reached a practical level (e.g. [2]). However, for outdoor navigation methods, dynamic changes in the environment still remains as a major problem. It is, therefore, necessary to strengthen the robustness of the outdoor navigation methods against changes in the surroundings.

We have proposed an outdoor navigation method called "MN (Magnetic Navigation) [3,4]". MN has robustness against moving objects since localization and control are performed by using only ambient magnetic field. We achieved the long distance run over $1 \mathrm{~km}$ as the mission of Tsukuba Challenge 2009 [5]. However, many trial runs and a lot of time ware required.

\section{黑 Springer}

C) 2014 Akai et al: licensee Springer. This is an Open Access article distributed under the terms of the Creative Commons Attribution License (http://creativecommons.org/licenses/by/2.0), which permits unrestricted use, distribution, and reproduction in any medium, provided the original work is properly credited. 
To solve this problem, we have proposed to use ambient geometric landmarks for compensating the robot's position (MNPC: Magnetic Navigation with Position Compensation [6]). The MNPC does not require many trial runs to achieve long distance navigation. However, in areas where magnetic anomalies occur, trajectory of the robot meanders. If magnetic anomalies occur in a wide range, the robot swerved from the desired path. It is a fatal problem since the MNPC uses a linear magnetic map, that contains only magnetic data on the desired path.

In this paper, we aim to improve the MNPC. For the improvement, we focused on a DCS (Distributed Control System). In the MNPC, there is one navigation module that controls the robot using magnetic sensor and LIDAR (Light Detection and Ranging) readings. If either magnetic sensor readings or LIDAR readings contains incorrect information, the control does not work well. However, precise navigation can be performed if sensor readings, which do not contain incorrect information, can be selected. Therefore, we divide the navigation module into several modules, and propose to use every module based on the DCS.

In this paper, we explain the proposed method and conduct two navigation experiments. Moreover, we took part in a guiding demonstration, which was held in Tsukubacity, Japan, during annual conference of ROBOMEC2013. By these results, the effectiveness and robustness of the proposed method are shown.

\section{Methods}

\section{Related works}

\section{Distributed control system}

Thus far, many types of DCS have been proposed. Brooks have proposed SA (Subsumption Architecture) [7]. Recently, SA is used as typical DCS. In SA, layers of control system are built to let a robot operate at increasing levels of competence. Rosenblatt have proposed DAMN (Distributed Architecture for Mobile Navigation) [8]. In DAMN, an arbiter performs command fusion and selects an action which best satisfies the prioritized goals of the system by voting. Morales et al. have proposed SSM (Sensor Sharing Manager) [9]. In SSM, many processes run in parallel such as localization, obstacle detection, and navigation. These DCSs allow the robot to generate robust and/or flexible behaviors.

In the proposed DCS, we focus on achieving a precise navigation. In our DCS, there are several modules and they generate the same behavior (trajectory tracking). Moreover, they respectively have a priority value, and this value is determined based on concordance rate of sensor readings and the database. The navigation module with the highest priority value is selected to control the robot. As the result, our robot can precisely navigate on the desired path.

\section{Autonomous navigation method}

Outdoor navigation methods using LIDARs or cameras have been proposed (e.g. [10,11]). Especially, localization technique based on ICP (Iterative Closest Point) algorithm [12] or MCL (Monte Carlo Localization) [13] is widely used since it allows a robot to precisely localize. However, since these sensors observe geometric landmarks, the localization accuracy can be easily affected by dynamic changes in its surroundings. In contrast, our proposed method allows the robot to stably navigate in dynamic outdoor environments even if LIDAR readings are used. It is because the DCS selects to use the MN instead of the navigation module using LIDAR readings.

As we mentioned in previous section, navigation methods using artificial magnetic landmarks have been proposed [1]. Bento et al. have proposed a navigation method using magnetic makers in semi-structure outdoor environment [14]. However, expensive initial cost is necessary in order to apply these method in wide outdoor environments. Moreover, these arrangements may spoil landscape of the city.

Localization technique using magnetic field, that occurs in natural environment, have been proposed. Suksakulchai et al. and Haverinen et al. have proposed localization technique using magnetic anomalies in indoor environment $[15,16]$. In these methods, linear maps are used for recording magnetic field and only the magnetic field on the desired paths were stored on the maps. However, these literatures do not present control technique for the robots. Since the maps do not have magnetic data other than on the path, swerving from the desired paths is a fatal problem.

Recently, localization technique using a 2D magnetic map has been proposed [17]. Frassl et al. achieved precise localization based on the 2D magnetic map [18]. However, constructing the $2 \mathrm{D}$ magnetic map in wide environments is difficult, namely adopting this technique to outdoor navigation is also difficult. Our method can achieve precise autonomous run even if localization is performed on the basis of the linear magnetic map.

An estimation method of the robot's heading direction using ambient magnetic field has been proposed [19]. However, as far as we know, none of these kinds of methods can achieve a long distance run. We then consider that this estimating method should be combined with another method to achieve a long distance run. In our method, localization technique using magnetic field and the control based on the DCS are combined.

In our method, since localization is performed by using the linear magnetic map, localization accuracy is not influenced by moving objects. However, since the map does not have enough information, it is difficult for the robot to precisely navigate on the desired path. Our 


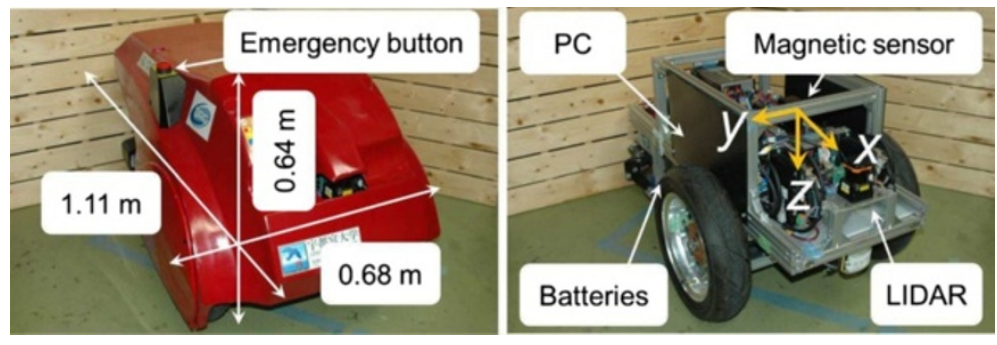

Figure 1 View of the robot with the armor (left) and without the armor (right).

method allows the robot to precisely navigate on the path by using DCS.

\section{Experimental platform}

The robot used as an experimental platform is shown in Figure 1. This robot has two encoders and they are mounted at the front wheels. A magnetic sensor (3DM$\mathrm{DH}$ ) and a LIDAR (UTM-30LX) are mounted on the robot. The magnetic sensor can measure three axes magnetic intensities $\left[b_{x} b_{y} b_{z}\right]$ and three magnetic azimuth angles $\left[\theta_{y} \theta_{r} \theta_{p}\right]$. Note that we only use $b_{z}$ and $\theta_{y}$ as magnetic sensor readings in this study. The $b_{z}$ is magnetic intensity scanned from the road surface, and $\theta_{y}$ is magnetic azimuth angle of horizontal plane. The scan range of the LIDAR is set to 180 degrees (the maximum scan range of the LIDAR is 270 degrees) since this LIDAR is mounted in the robot's armor.

\section{Magnetic navigation with position compensation}

The basic idea of localization technique of the MNPC is shown in Figure 2. A linear magnetic map is used and it records ambient magnetic field on a desired path as magnetic information $\boldsymbol{M}$. The information is recorded according to the travel distance. In actual environment, there are magnetic anomalies produced by magnetized material (e.g. manholes). These anomalies can be used as landmarks since they are stable in time scale. The robot fixes a travel distance by using the landmarks [3,4]. Moreover, a heading direction of the robot is estimated by using stable magnetic field.

Table 1 shows an example of the database. It is constructed by manually operating the robot before autonomous navigation. Each data is recorded according to the travel distance and data recording interval is $10 \mathrm{~cm}$. Magnetic sensor readings are directly used as the magnetic information $\boldsymbol{M}$, and the information recorded in node $n$ is denoted as $\boldsymbol{m}_{n}=\left[b_{z, n} \theta_{y, n}\right]$.

Figure 3 shows how the LIDAR is used in the MNPC. Scan ranges are set in front left and right of the robot. The length of these ranges $R$ is $6 \mathrm{~m}$ and the angle $\alpha$ is 30 degrees. The reason of which these ranges are set at the sides is that the LIDAR is used as compensating a lateral error from the desired path. The minimum lateral lengths measured in each range are used as geometric information $\boldsymbol{G}$, and they are denoted as $g_{l}$ and $g_{r}$. The information recorded in node $n$ is denoted as $\boldsymbol{g}_{n}=\left[g_{l, n} g_{r, n}\right]$.

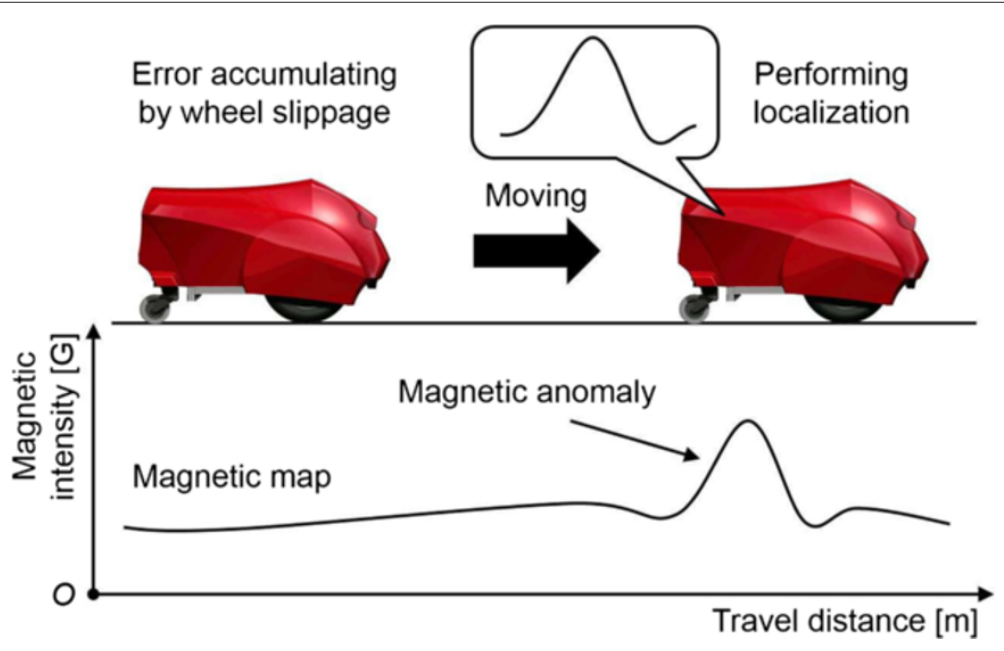

Figure 2 Conceptual figure of the localization technique on magnetic navigation with position compensation. 
Table 1 An example of the database used on the MNPC

\begin{tabular}{cccc}
\hline $\begin{array}{c}\text { Travel } \\
\text { distance }\end{array}$ & $\begin{array}{c}\text { Heading } \\
\text { direction }\end{array}$ & $\begin{array}{c}\text { Magnetic } \\
\text { information } \boldsymbol{M}\end{array}$ & $\begin{array}{c}\text { Geometric } \\
\text { information } \boldsymbol{G}\end{array}$ \\
\hline$d_{0}$ & $\theta_{0}$ & $\boldsymbol{m}_{0}$ & $\boldsymbol{g}_{0}$ \\
\hline$d_{1}$ & $\theta_{1}$ & $\boldsymbol{m}_{1}$ & $\boldsymbol{g}_{1}$ \\
\hline$\vdots$ & $\vdots$ & $\vdots$ & $\vdots$ \\
\hline$d_{n}$ & $\theta_{n}$ & $\boldsymbol{m}_{n}$ & $\boldsymbol{g}_{n}$ \\
\hline
\end{tabular}

The MNPC controls the robot as converging all components of $\boldsymbol{e}_{c}$ to zero by controlling robot's angular velocity $\omega$.

We achieved outdoor autonomous navigation over $2 \mathrm{~km}$ by using the MNPC [6]. However, the MNPC has a fatal problem.

\section{A fatal problem of the MNPC}

As we mentioned in the previous section, swerving from the desired path is a fatal problem when using the linear magnetic map. However, the trajectory easily meanders in an area with magnetic anomalies since the control using magnetic sensor readings does not work well. As the result, the robot easily swerves from the path. In MNPC, geometric information is used for compensating an error produced by the meander. However, if ambient geometric information is also dynamically changed, the compensation does not work well. This means that it is difficult to adopt the MNPC in dynamic environment navigation.

Avoiding moving objects, which intersect the desired path, is also fatal problem since the robot swerves from its desired path. However, if the robot can precisely navigate on its desired path, avoiding moving objects is not necessary. Therefore, in our navigation strategy, the robot will stop when an obstacle appears in front of it.

\section{Proposed method}

The key idea of our proposed method is to use only effective sensor readings for robot's control. The MNPC has three different information of magnetic sensor, LIDAR, and encoders readings. The quality of these information differs from each others. For instance, there is a case where LIDAR readings are not containing incorrect information even if magnetic sensor readings are containing incorrect information. In this case, meandering the trajectory can be prevented if LIDAR readings can be only used for the control. Therefore, we divide the navigation module of the MNPC into several modules, and propose to use every module based on the DCS.

Figure 4 shows the system scheme of the proposed method. Localization is performed by using magnetic information $\boldsymbol{M}$, magnetic sensor readings $\boldsymbol{m}_{t}$, and encoder readings $\boldsymbol{a}_{t}$. A state of the robot at time $t \boldsymbol{X}_{t}$ is expressed by a travel distance $d_{t}$ and a heading direction $\theta_{t}$.

The robot localizes its own travel distance $d$ and heading direction $\theta$ by using only the magnetic information $\boldsymbol{M}$. Currently, the robot is locating at node $n$ and observing magnetic information $\boldsymbol{m}_{t}$ and geometric information $\boldsymbol{g}_{t}$, the deflections used for robot's control $\boldsymbol{e}_{c}$ are determined as follow:

$$
\boldsymbol{e}_{c}=\left[\theta_{y, n}-\theta_{y, t} \quad g_{l, n}-g_{l, t} \quad g_{r, t}-g_{r, n}\right] .
$$

There are several navigation modules in the system and each module called a "navigator". Each navigator is defined to navigate the robot based on each sensor readings. Each navigator independently send a control input $\boldsymbol{u}_{i}(i=1,2, \ldots, k)$ and a priority $p_{i}$ to the management module. This management module then selects an actual control input $\boldsymbol{u}$ based on the priority value, which is determined by comparing external sensor readings $\left(\boldsymbol{m}_{t}\right.$ and $\left.\boldsymbol{g}_{t}\right)$ with the database $\left(\boldsymbol{m}_{n}\right.$ and $\left.\boldsymbol{g}_{n}\right)$. The $\boldsymbol{g}_{t}$ is geometric information observed at time $t$. If sensor readings are unreliable, the priority value of the navigator using

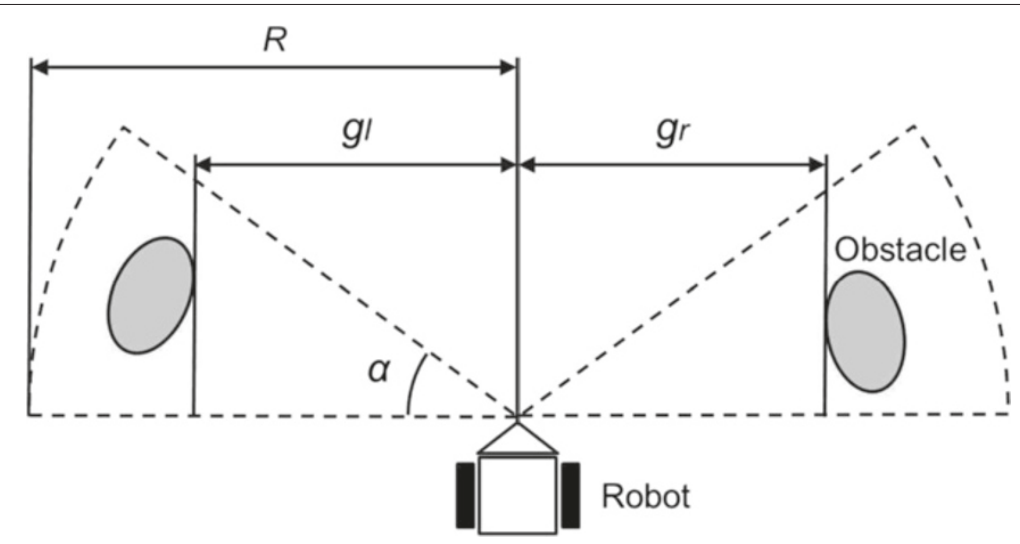

Figure 3 Geometric landmark information detected by LIDAR. 


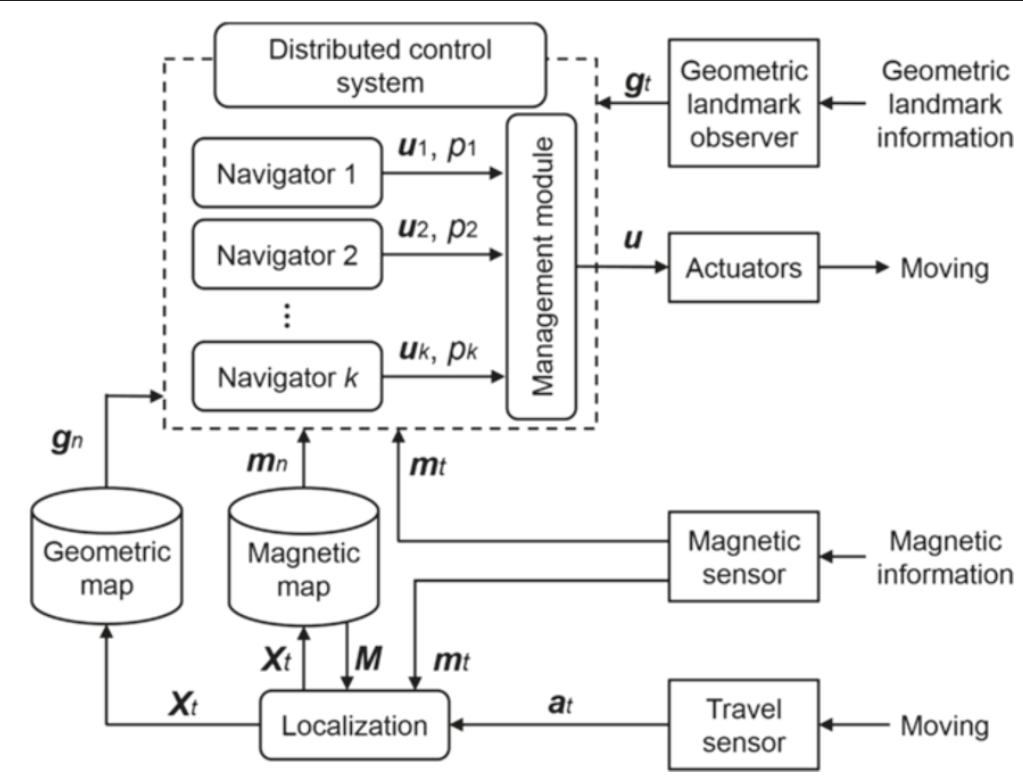

Figure 4 System figure of the proposed method.

those sensor readings is set to a low value. With this method, an reliable navigator can be selected.

The configuration our DCS is shown in Figure 5. Several navigators are executed in parallel. All the sensor readings are temporarily stored in the shared memory, which can allow all the navigators to access to. In this study, we used four navigators; (1) navigator based on odometry, (2) navigator based on geometric information, (3) navigator based on magnetic information, and (4) navigator based on magnetic and geometric information (MNPC).

\section{Navigator based on odometry}

If the robot is locating at node $n$, and $\theta_{t}$ is its actual heading direction, the deflection $e_{o}$ can be determined as follow:

$$
e_{o}=\theta_{n}-\theta_{t}
$$

The navigator converges this deflection to zero by controlling the angular velocity.

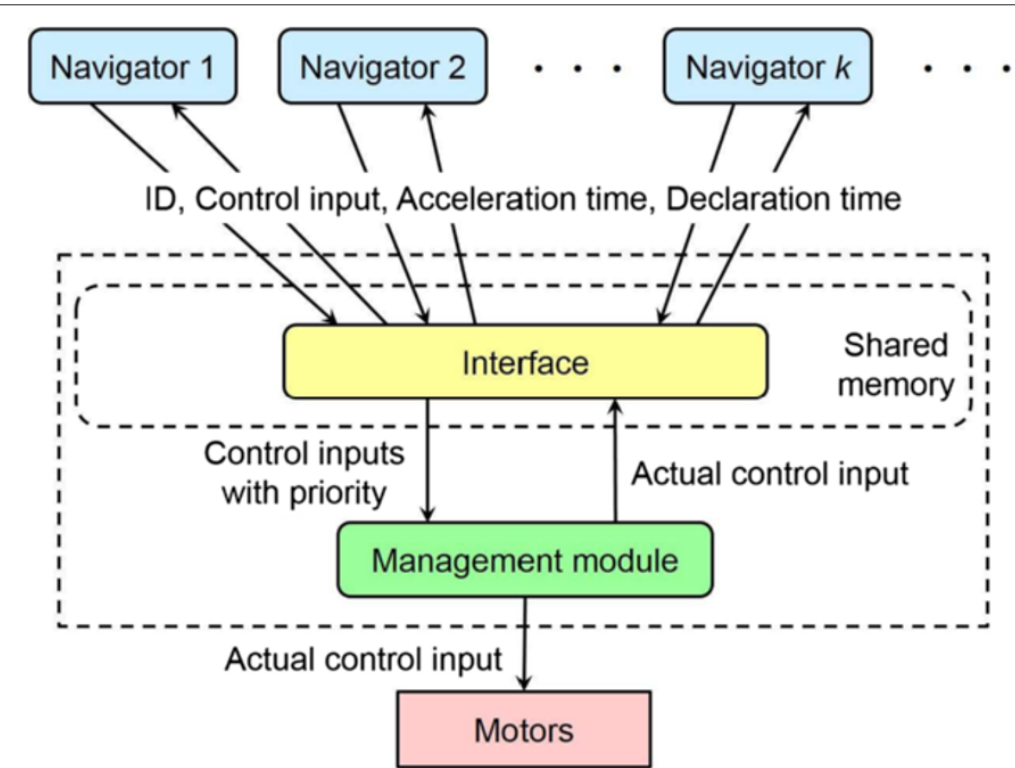

Figure 5 Configuration figure of the distributed control system. 


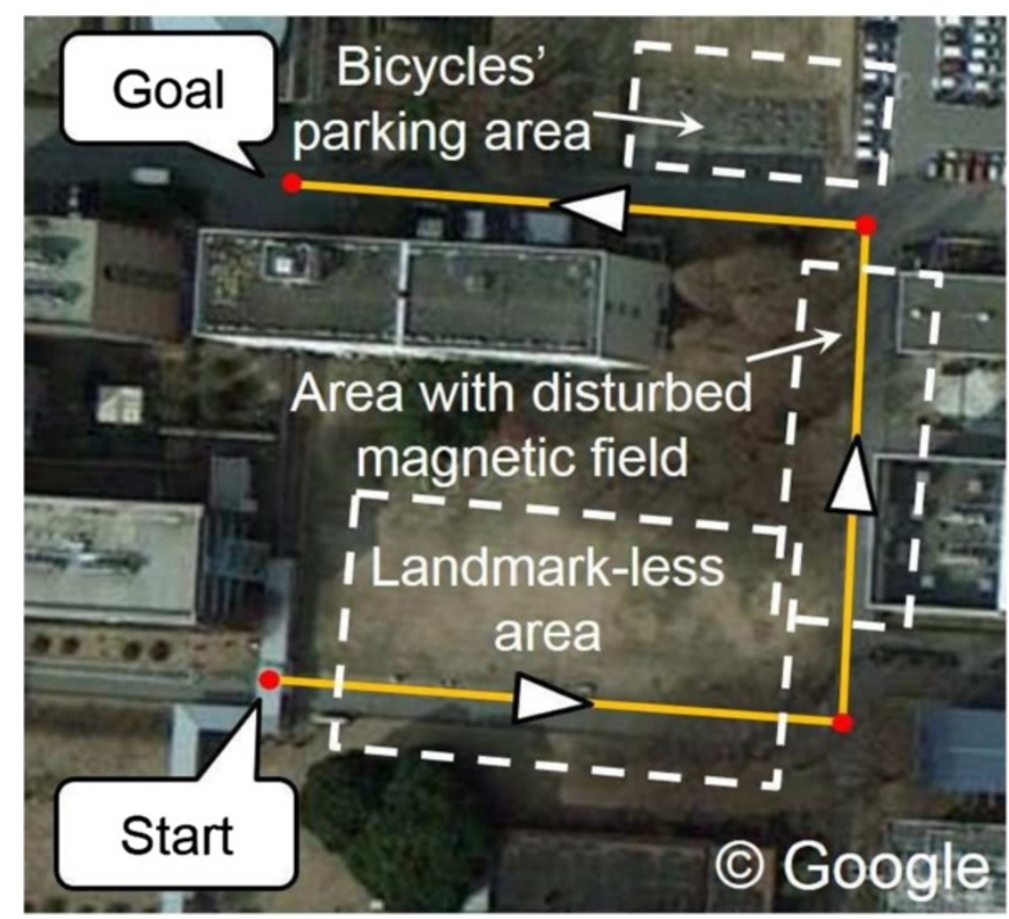

Figure 6 Overview of the experimental course.

\section{Navigator based on geometric information}

If the robot currently locates at node $n$ and a geometric information $\boldsymbol{g}_{t}=\left[g_{l, t} g_{r, t}\right]$ is observed, the deflection $\boldsymbol{e}_{l}$ can be determined as follow:

$$
\boldsymbol{e}_{l}=\left[\begin{array}{lll}
g_{l, n}-g_{l, t} & g_{r, t}-g_{r, n} & \theta_{n}-\theta_{t}
\end{array}\right] .
$$

The navigator converges all components of $\boldsymbol{e}_{l}$ to zero by controlling the angular velocity.

\section{Navigator based on magnetic information}

If the robot is locating at node $n$ and $\boldsymbol{m}_{t}=\left[b_{z, t} \theta_{y, t}\right]$ is a magnetic information measured by the magnetic sensor, the deflection $e_{m}$ can be determined as follow:

$$
e_{m}=\theta y, n-\theta y, t \text {. }
$$

The navigator converges this deflection to zero by controlling the angular velocity.

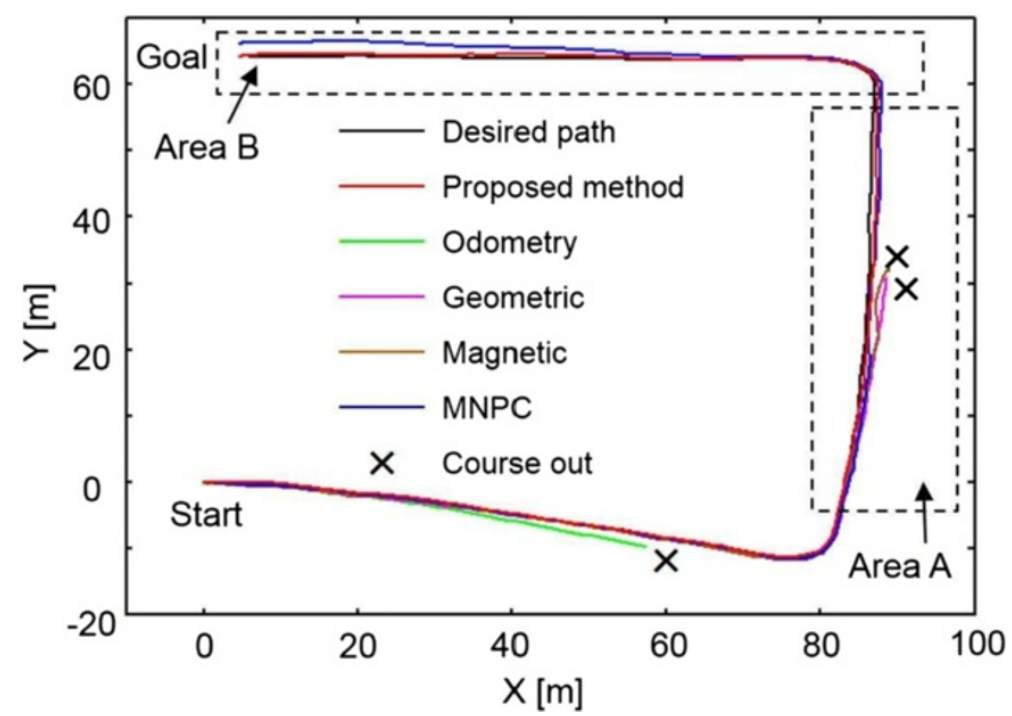

Figure 7 Trajectories of each navigation method. 
Table 2 Trajectory tracking errors from the desired path

\begin{tabular}{cccccc}
\hline & Proposed method & MNPC & Magnetic & Geometric & Odometry \\
\hline Average $[\mathrm{m}]$ & 0.171 & 0.673 & 0.481 & 0.479 & 0.720 \\
\hline Standard deviation $[\mathrm{m}]$ & 0.130 & 0.686 & 0.586 & 0.643 & 0.620 \\
\hline
\end{tabular}

\section{Navigator based on magnetic and geometric information}

This navigator uses magnetic and geometric information, namely it is the MNPC. The detail of the MNPC is mentioned above.

\section{Priority determination}

A priority value of each navigator $p_{i}$ is determined as follow:

$$
p_{i}= \begin{cases}\alpha_{i} & (\text { used sensor readings are reliable }) \\ 0 & (\text { used sensor readings are unreliable })\end{cases}
$$

where $\alpha_{i}$ are plus fixed numbers. We set a high fixed number to a navigator, which can precisely navigate the robot. The order of the priority vales is as following; (1) the MNPC (2) navigator based on magnetic information (3) navigator based on geometric information (4) navigator based on odometry. It was defined on the basis of our experiences.

In a case where moving objects across nearby the robot, the navigator using geometric information does not work well. The reliability of LIDAR readings is determined by using these difference values $\left|g_{l, n}-g_{l, t}\right|$ and $\left|g_{r, t}-g_{r, n}\right|$. If the values exceed $1.0 \mathrm{~m}$, the priority of the MNPC and the navigator based on geometric information become zero.

In a case where the magnetic sensor measures noise, navigator using magnetic information does not work well.
The reliability of magnetic sensor readings is determined by using a variable $p_{m}$, which is expressed as follow:

$$
p_{m}=1-\frac{\left|b_{z, n}-b_{z, t}\right|}{\Delta b_{\max }}
$$

where $\Delta b_{\max }$ is a difference value of maximum and minimum magnetic intensity $b_{z}$ measured by used magnetic sensor. In this study, we defined $\Delta b_{\max }=1$ on the basis of our experiences. Magnetic sensor readings are reliable when the value of $p_{m}$ closes to 1 . If $p_{m}$ is less than 0.998 , the priority value of the the MNPC and the navigator based on magnetic information become zero.

\section{Results and discussion}

\section{Navigation experiment}

We conducted a navigation experiment for verifying the effectiveness of the proposed method by comparing five navigation methods; the proposed method and four navigators in the proposed method. Note that the experiment was conducted after three days of database construction.

Figure 6 shows the overview of the experimental environment. In this environment, there are some difficult areas for navigation; landmark-less area, bicycles' parking area, and area with disturbed magnetic field. The trajectories by each method are shown in Figure 7. The trajectories were obtained by using our localization method [20], and obviously outlier was manually corrected. As can

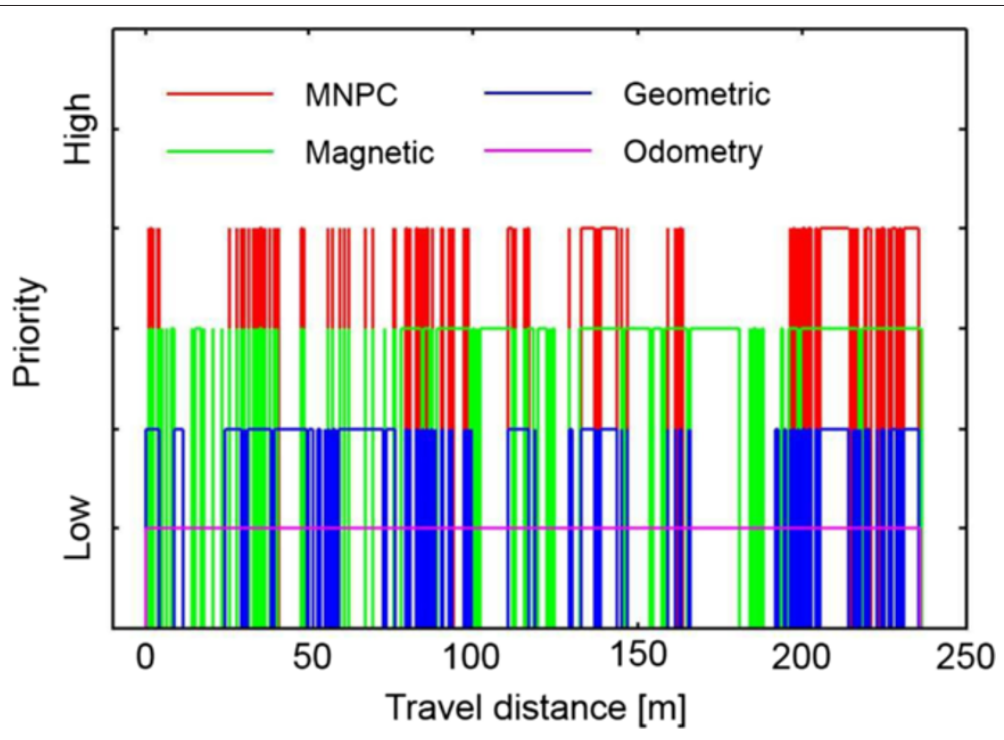

Figure 8 Priorities of each navigator in the proposed method. 


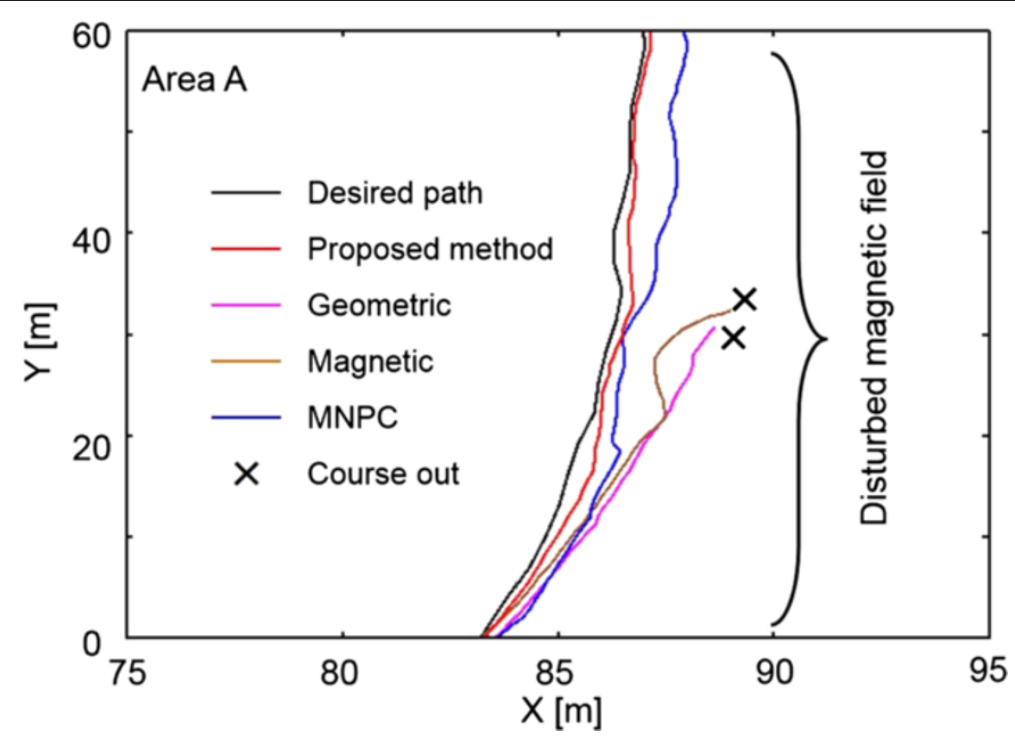

Figure 9 Trajectories in Area A in Figure 7.

be seen from Figure 7, only the proposed method and the MNPC could complete the autonomous navigation. Table 2 shows average and standard deviation of trajectory tracking error of each method. From the table, we could confirm that the proposed method achieved the most precise navigation.

Figure 8 shows priorities of each navigator in the proposed method. These priorities were dynamically changed. By combining the results of Figure 8 and Table 2, it showed that the control method based on the DCS effectively performed in the environment and provided the robot a precise navigation.
Figure 9 and Figure 10 are enlarged figures of Area A and $B$ in Figure 7. In Area A, the trajectory of the navigator using magnetic information meandered since the magnetic field was disturbed. Moreover, in Area B, the trajectory of the MNPC swerved since state of the bicycles' parking area was changed from the time of database construction. Even if the proposed method used both of magnetic and geometric information, precise navigation could be conducted. This means that the MNPC was improved by using the DCS.

Moreover, Figure 11 shows magnetic intensity on the desired path (red line) and value of $p_{m}$ determined by

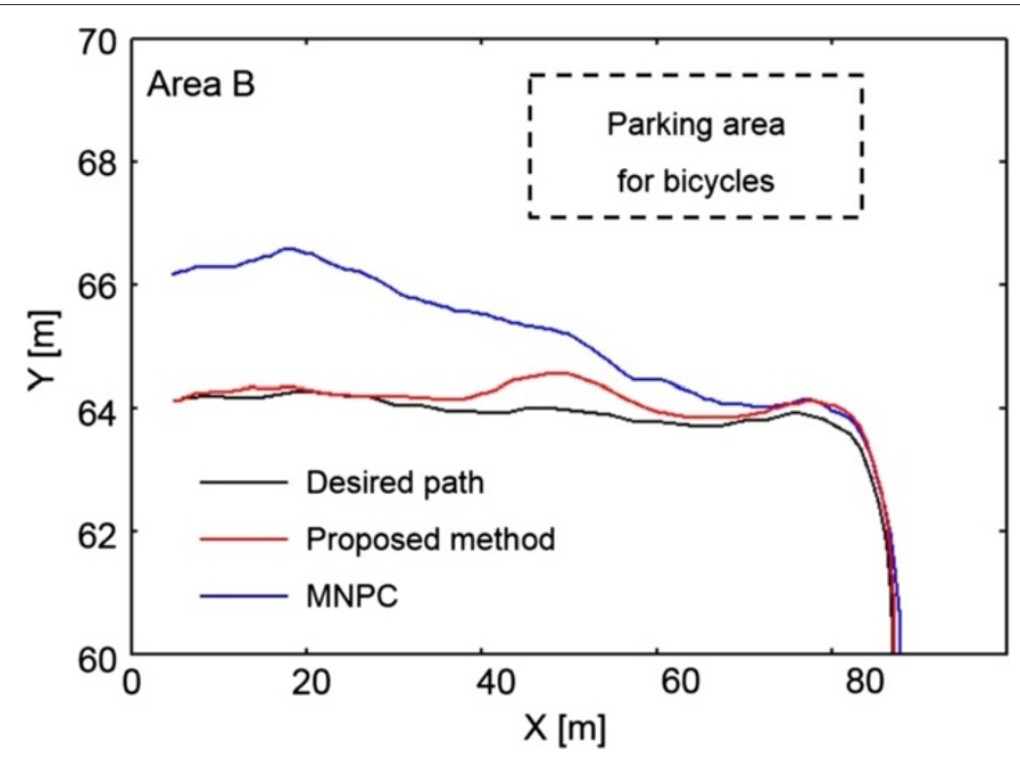

Figure 10 Trajectories in Area B in Figure 7. 


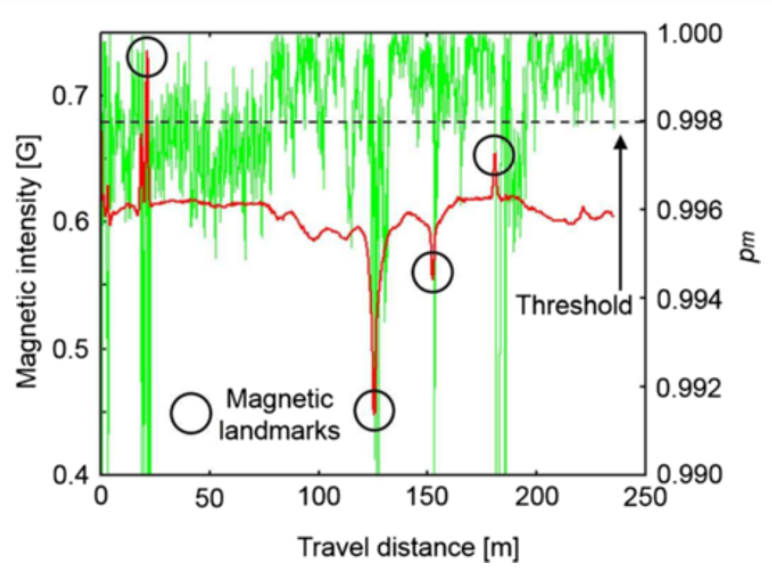

Figure 11 Magnetic intensity on the desired path (red line) and reliability (green line) obtained in autonomous navigation.

Eq. (6) (green line). The value of $p_{m}$ decreased around magnetic anomalies, which were used as magnetic landmarks and some other areas. This shows that the variable $p_{m}$ effectively worked in the proposed method. From these results, the effectiveness of the proposed method was shown.

\section{Simulated guiding demonstration}

We conducted a simulation experiment of the guiding demonstration in our campus. Figure 12 shows the experimental scenario. The robot ran on the desired path from left to right and a guidance target followed by walking behind of the robot. In addition, three groups of pedestrians passed by the robot as shown by the yellow arrows.

Figure 13 shows the result of the experiment. The robot stably navigated on the desired path even if the three groups walked around the robot. When the groups passed nearby the robot, the navigators using geometric information did not work and the robot was controlled by the navigator based on magnetic information or the navigator based on odometry. As the result, the robot did not meander and the navigation was not influenced by the moving objects.

After the groups walked away, the robot used geometric information for the lateral error compensation and a stable navigation was restored. The experimental result shows that the proposed method can be adopted to navigation in a busy pedestrian walkway.

\section{Guiding demonstration in ROBOMEC2013}

The guiding demonstration using autonomous mobile robots was held in Tsukuba-city, Japan, and we took part in this demonstration. In this demonstration, robots should lead persons from the start area to the destination in actual busy pedestrian walkway.

The views of this demonstration are shown in Figure 14. Figure 14(a),(b) show a case where many children were gathered in front of the robot and they walked with the robot. Moreover, the case where many pedestrian come

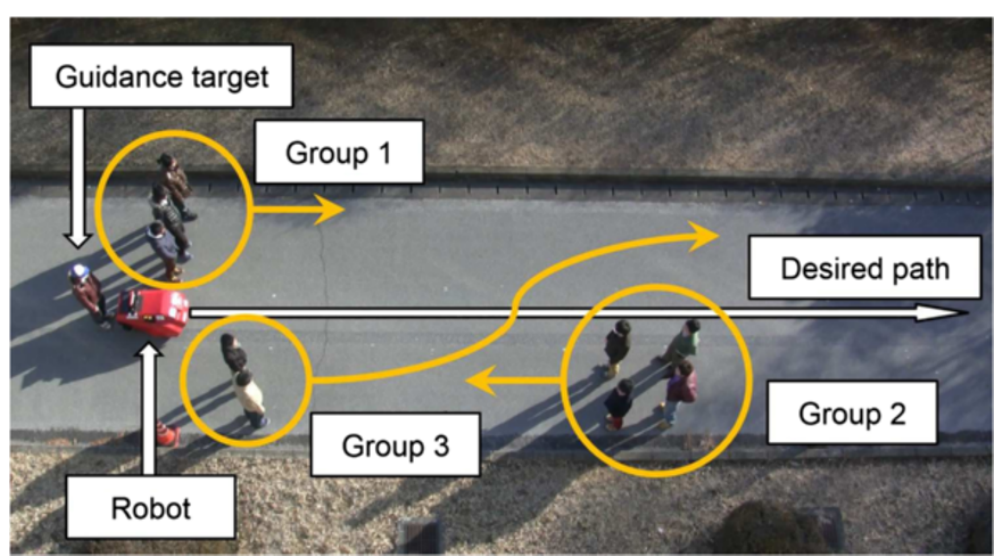

Figure 12 An experimental scenario of the simulated guiding demonstration. 


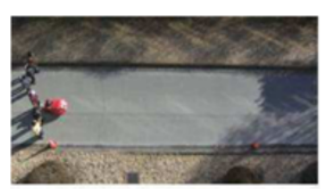

a $t=0.0 \mathrm{~s}$

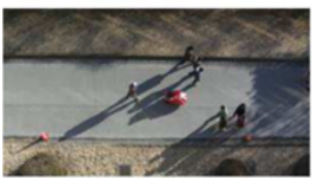

f $t=10.0 \mathrm{~s}$

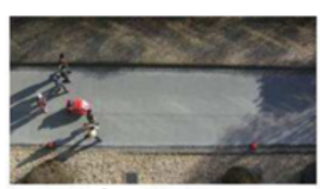

b $t=2.0 \mathrm{~s}$

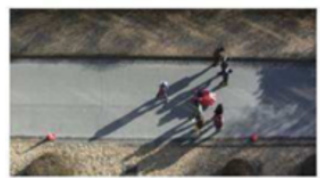

$\mathrm{g} t=12.0 \mathrm{~s}$

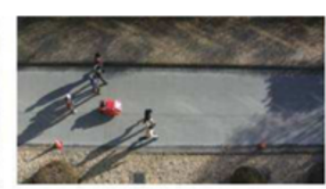

C $t=4.0 \mathrm{~s}$

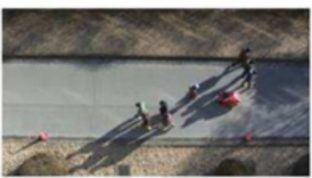

h $t=14.0 \mathrm{~s}$

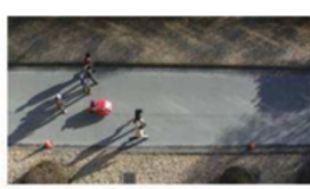

$\mathrm{d} t=6.0 \mathrm{~s}$

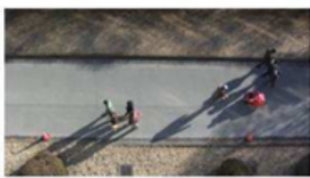

i $t=16.0 \mathrm{~s}$

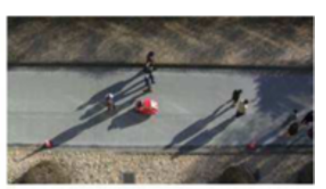

e $t=8.0 \mathrm{~s}$

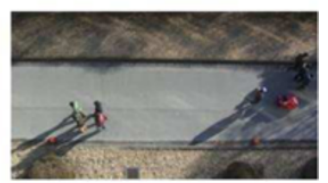

j $t=18.0 \mathrm{~s}$

Figure 13 Experimental result of the simulated guiding demonstration.

and go around the robot often occurred (see Figure 14(c)). These cases were similar to the experiment, which we conducted in our campus. In these cases, the navigator based on magnetic information or the navigator based on odometry were performed. As the result, stable navigation could be performed even if available geometric landmarks could not be observed.

Figure 14(d) shows an interesting case where there was a truck nearby the desired path. The truck influenced magnetic field and blocked detection of available geometric landmarks. In this case, the navigator based on odometry was performed. The robot ran approximately $10 \mathrm{~m}$ and achieved autonomous navigation. Autonomous navigation based on odometry is usually unsuitable, however, in this case, it is suitable since there are no effective information. Our method could select navigator based on odometry and realized stable navigation in the dynamic environment.

In one demonstration, the robot ran approximately $700 \mathrm{~m}$. We conducted 27 guiding demonstrations and our robot could perfectly accomplished all demonstrations. From these results, the robustness of the proposed method was confirmed.

\section{Conclusions}

In this paper, we proposed a navigation method for improving the MNPC. The improvement could be fulfilled by using the DCS. In our method, since the linear magnetic map is used for localization, swerving from a desired path is fatal problem. Our proposed method could solve

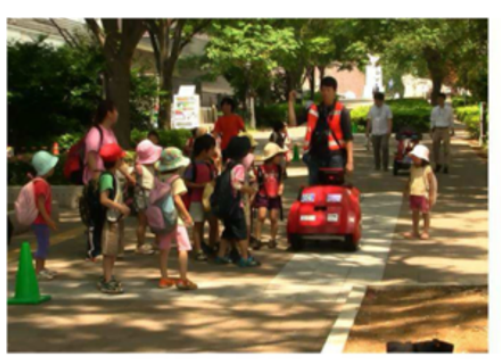

a

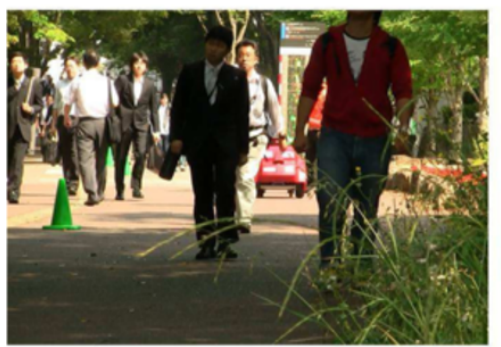

C

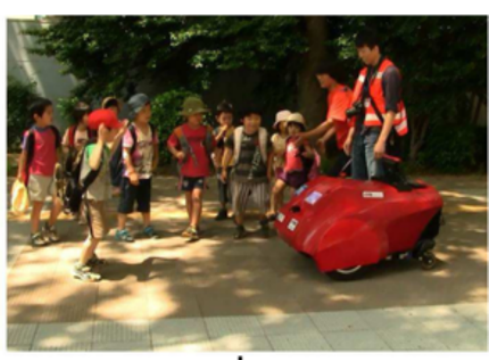

b

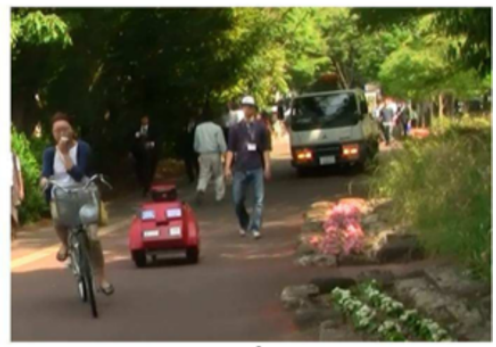

d

Figure 14 Views of the guiding demonstration. (a) Children gathered near the robot. (b) A child stood on the robot's way. (c) Pedestrians walked around the robot. (d) New magnetized material was appeared. 
the problem and achieved precise autonomous navigation in dynamic outdoor environments, since used DCS can select the navigator, which precisely navigates the robot on the desired path. We took part in the guiding demonstration held in the central of Tsukuba-city, Japan. In the demonstration, we conducted 27 guiding, and miss guiding was not done. From these results, we showed the effectiveness and robustness of the proposed method.

\section{Competing interests}

The authors declare that they have no competing interests.

\section{Authors' contributions}

NA and SAR proposed the navigation method. NA and $\mathrm{KI}$ are members of the developing team. NA, Kl, and $\mathrm{KO}$ conducted the experiments. All authors have read and approved the final manuscript.

\section{Acknowledgements}

This study was supported by KAKENHI (25420210). Moreover, the first author was supported by Mr. Saito.

\section{Author details}

${ }^{1}$ Department of Innovation System Engineering, 7-1-2, Yoto, Utsunomiya-shi, Tochig, Japan. ${ }^{2}$ Department of Electrical and Information Engineering, Oyama National College of Technology, 771, Nakakuki, Oyama-shi, Tochigi, Japan.

Received: 14 January 2014 Accepted: 20 June 2014

Published online: 21 November 2014

\section{References}

1. Lee SY, Yang HW (2012) Navigation of automated guided vehicles using magnet spot guidance method. Robot Comput-Integr Manuf 28(3):425-436

2. Maaref H, Barret C (2002) Sensor-based navigation of a mobile robot in an indoor environment. Robot Autonomous Syst 38:1-18

3. Rahok SA, Ozaki K (2008) Localization system based on magnetic map for indoor mobile robot. In: JSME conference on robotics and mechatronics. Nagano, Japan, 5-7 June 2008 (in Japanese)

4. Rahok SA, Shikanai Y, Ozaki K (2011) Navigation using an environmental magnetic field for outdoor autonomous mobile robots. Adv Robot 25(13-14):1751-1771

5. Nagatani K, Kushleyev A, Daniel DL (2012) Sensor information processing in robot competitions and real world robotic challenge. Adv Robot 26(14):1539-1554

6. Akai N, Rahok SA, Inoue K, Ozaki K (2013) Implementation of a long-distance navigation method of low cost structure that combines a localization using magnetic information and a lateral position compensation. Trans Japan Soc Mech Eng 79(799):681-690 (in Japanese)

7. Brooks RA (1986) A robust layered control system for a mobile robot. IEEE J Robot Automation 2(1):14-23

8. Rosenblatt JK (1997) DAMN: a distributed architecture for mobile navigation. J Exp Theor Artif Intell 9(2-3):339-360

9. Morales Y, Carballo A, Takeuchi E, Aburadani A, Tsubouchi T (2009) Autonomous robot navigation in outdoor cluttered pedestrian walkways. J Field Robot 26(8):609-635

10. Yoshida T, Irie K, Koyanagi E, Tomono M (2010) A sensor platform for outdoor navigation using gyro-assisted odometry and roundly-swinging 3D laser scanner. In: International conference on intelligent robots and systems, Taipei, Taiwan, 18-22 October 2010

11. Irie K, Yoshida T, Tomono M (2012) Outdoor localization using stereo vision under various illumination conditions. Adv Robot 26(3-4):327-348

12. Chen Y, Medioni G (1991) Object modeling by registration of multiple range images. In: International conference on robotics and automation, California, United States, 9-12 April 1991

13. Dellaert F, Fox D, Burgard W, Thrun S (1999) Monte Carlo localization for mobile robots. In: International conference on robotics and automation, Michigan, United States, 10-15 May 1999
14. Bento LC, Nunes U, Moita F, Surrecio A (2005) Sensor fusion for precise autonomous vehicle navigation in outdoor semi-structured environments. In: International conference on intelligent transportation systems. Congress Center MesseWienNeuVienna, Austria, 13-16 September 2005

15. Suksakulchai S, Thongchai S, Wilkes DM, Kawamura K (2000) Mobile robot localization using an electronic compass for corridor environment. In: International conference on systems, man and cybernetics, Nashville, United States, 8-11 October 2000

16. Haverinen J, Kemppainen A (2009) Global indoor self-localization based on the ambient magnetic field. Robot Autonomous Syst 57:1028-1035

17. Gozick B, Subbu KP, Dantu R, Maeshiro T (2011) Magnetic maps for indoor navigation. IEEE Trans Instrum Meas 60(12):3883-3891

18. Frassl M, Angermann M, Lichtenstern M, Robertson P, Julian BJ, Doniec M (2013) Magnetic maps of indoor environments for precise localization of legged and non-legged locomotion. In: International conference on intelligent robots and systems, Tokyo, Japan, 3-8 November 2013

19. Woong K, Roh KS, Sung HK (2006) Particle filter-based heading estimation using magnetic compasses for mobile robot navigation. In: International conference on robotics and automation, Florida, United States, 15-19 May 2006

20. Akai N, Inoue K, Ozaki K (2014) Autonomous navigation based on magnetic and geometric landmarks on environmental structure in real world. J Robot Mechatronics 26(2):158-165

doi:10.1186/s40648-014-0021-8

Cite this article as: Akai et al:: Development of magnetic navigation method based on distributed control system using magnetic and geometric landmarks. ROBOMECH Journal 2014 1:21.

\section{Submit your manuscript to a SpringerOpen ${ }^{\odot}$ journal and benefit from:}

- Convenient online submission

Rigorous peer review

- Immediate publication on acceptance

- Open access: articles freely available online

- High visibility within the field

- Retaining the copyright to your article

Submit your next manuscript at $>$ springeropen.com 